\title{
Relationship between different experimental measures of distorted symptom perception in functional syndrome patients
}

Running head: symptom perception in FSS patients

Maaike Van Den Houte ${ }^{\mathrm{a}, \mathrm{b}, \mathrm{d}}, \mathrm{PhD}$, Lukas Van Oudenhovec ${ }^{\mathrm{c}}, \mathrm{MD}, \mathrm{PhD}$, Katleen Bogaerts ${ }^{\mathrm{a}, \mathrm{d}}, \mathrm{PhD}$, Ilse Van Diest ${ }^{\mathrm{a}}$, PhD, Jozef De Bie ${ }^{\mathrm{f}}, \mathrm{MD}$, Philippe Persoons ${ }^{\mathrm{e}}, \mathrm{MD}, \mathrm{PhD}, \&$ Omer Van den Bergh $^{\mathrm{a}}, \mathrm{PhD}$

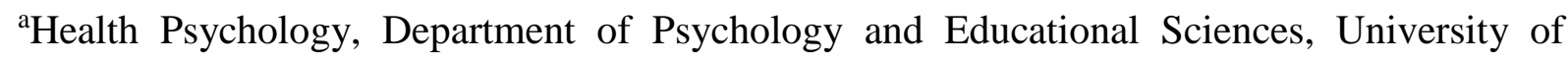
Leuven, Belgium

${ }^{\mathrm{b}}$ Mind-Body Research, Department of Neurosciences, University of Leuven, Belgium

${ }^{\mathrm{c}}$ Laboratory for Brain-Gut Axis Studies (LaBGAS), Translational Research Center for Gastrointestinal Disorders (TARGID), University of Leuven, Belgium.

${ }^{\mathrm{d} R E V A L ~-~ R e h a b i l i t a t i o n ~ R e s e a r c h ~ C e n t e r, ~ F a c u l t y ~ o f ~ R e h a b i l i t a t i o n ~ S c i e n c e s, ~ H a s s e l t ~}$ University, Diepenbeek, Belgium

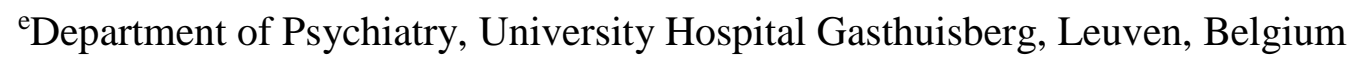

${ }^{\mathrm{f}}$ Centre for Translational Psychological Research (TRACE), Hospital ZOL Limburg, Genk, Belgium

Corresponding author:

Maaike Van Den Houte, PhD

KULeuven - University of Leuven / Hasselt University

maaike.vandenhoute@kuleuven.be / maaike.vandenhoute@uhasselt.be

Agoralaan Gebouw A - 3590 Diepenbeek, Belgium

Tel: +32(0)11269305 - Fax: +32(0)11269376

Number of words: 6294

Number of tables: 2

Number of figures: 2

Conflicts of Interest and Source of Funding: The authors report no conflicts of interest. This research was funded by the Center for Excellence on Generalization Research (GRIP*TT; University of Leuven grant $\mathrm{PF} / 10 / 005$ ) and by Asthenes, a long-term structural fundingMethusalem grant by the FWO-Vlaanderen, Flemish Government, Belgium. The study was 
further supported by infrastructure grants from the Herculesstichting, Belgium (AKUL/13/07 and AKUL/11/08).

\section{$\underline{\text { Abstract }}$}

Objective: Patients with functional somatic syndromes (FSS) show reduced correspondence between induced physiological changes and self-reported symptoms in a rebreathing paradigm, as well as elevated symptoms unrelated to physiological changes after induction of negative affective states in an affective picture viewing paradigm. Detailed results of both paradigms separately were published elsewhere. The main goal of the current report is to describe the relationship between the responses to these two paradigms measuring distortions in symptom perception in a well-described sample of patients with fibromyalgia and/or chronic fatigue syndrome (CFS).

Methods: Patients $(\mathrm{N}=81)$ with fibromyalgia and/or CFS participated in a test session comprising four well-validated paradigms, including the picture viewing and rebreathing paradigm. Using mixed model analyses, it was tested whether the amount of affective modulation of symptom reporting was related to distorted perception of induced dyspnea. In an exploratory way, we assessed the role of several individual difference variables as moderators.

Results: There was no relationship between patients' amount of affective modulation of symptom reporting, as assessed with the picture paradigm, and level of distortion in dyspnea perception, as assessed with the rebreathing paradigm (effect of affective modulation in the subjective recovery from induced dyspnea: $F_{1,70}=0.16, p=0.70$; time*affective modulation interaction effect: $\left.\mathrm{F}_{4,70}=0.14, \mathrm{p}=0.97\right)$.

Conclusions: Biased symptom reporting in one paradigm is unrelated to biased symptom reporting in the other paradigm, indicating that distortions in symptom perception in FSS patients is not a trait-like, cross-situationally stable condition, but a versatile dysfunction that is context-dependent. 
Key words: symptom perception, fibromyalgia, chronic fatigue syndrome, interoception, dyspnea, emotion regulation

\section{List of abbreviations:}

ACR: American College of Rheumatology

CDC: Center for Disease Control and Prevention

CFS: Chronic Fatigue Syndrome

$\mathrm{FetCO}_{2}$ : Fractional end-tidal levels of carbon dioxide

FSS: Functional Somatic Syndromes

HSR: Habitual Symptom Reporters

IAS: Illness Attitude Scales

IAQ: Interoceptive awareness questionnaire

MINI: MINI International Neuropsychiatric Interview

PANAS: Positive and Negative Affect Schedule

$\mathrm{PCO}_{2}$ : Partial pressure of carbon dioxide in the blood

PHQ: Patient Health Questionnaire

TAS-20: Toronto Alexithymia Scale 


\section{Introduction}

Functional somatic syndromes (FSS) such as fibromyalgia or chronic fatigue syndrome (CFS) are characterized by chronic and severe physical symptoms insufficiently explained by organic dysfunction. Despite intensive research over the past decades, the etiology and pathogenesis of these disorders is still unclear, but generally, complex interactions between biological, psychological and social mechanisms are believed to cause and exacerbate the symptoms (1). In this regard, the importance of interoceptive and symptom perception processes in the development and maintenance of functional symptoms and FSS is increasingly acknowledged. Interoception refers to the "processing of internal bodily stimuli by the nervous system" (2), while symptom perception refers to perception of bodily dysfunction, thus awarding internal bodily stimuli negative value and interpreting them as being a potential threat to the healthy body. Before reaching consciousness, afferent signals from the body are modulated by interacting bottom-up and top-down processes, such as attentional, affective and cognitive processes, to ensure that individuals perceive what is relevant to them in a specific context (3). Therefore, the correspondence between perceived symptoms and bodily dysfunction varies between persons and within persons between different contexts. It has been proposed that FSS are at least partly disorders of symptom perception, where the afferent signals become modulated in such a way that the perceived symptoms are only loosely related to bodily dysfunction. More specifically, in situations where afferent sensory-perceptual information is low in detail, the brain may rely more heavily on top down-modulation driven by prior information/expectations when interpreting interoceptive input $(4,5)$.

Several studies from our group have demonstrated that healthy individuals with high levels of somatic symptoms (high habitual symptom reporters; HSR) and clinical FSS patients process and experience experimentally induced symptoms differently than low HSR/healthy controls (for an overview, see 6), especially in negative affective contexts. For instance, it has 
consistently been shown with a picture viewing paradigm that symptoms can be induced in high HSR and patients with irritable bowel syndrome by the mere presentation of negative affective cues (7-9): when presented with a symptom checklist, high HSR and FSS patients reported more somatic symptoms after viewing negative (symptom-related or not symptom-related) compared to neutral or positive pictures, despite the groups being similar in physiological arousal. Second, high HSR and FSS patients show a reduced correspondence between experimentally induced physiological changes and self-reported symptoms. This was for instance investigated in a rebreathing paradigm, where a progressive increase in self-reported dyspnea/air hunger, increased respiratory flow and hypercapnia (excessive levels of $\mathrm{CO}_{2}$ in the blood) is induced by letting participants rebreathe through a bag initially filled with a mixture of $\mathrm{CO}_{2}$ and oxygen. A series of studies from our group using this technique has shown that the correspondence between subjective dyspnea and respiratory flow, and subjective dyspnea and end-tidal $\mathrm{CO}_{2}$ levels, is lower in high HSR and in patients with medically unexplained dyspnea, but only when the paradigm was presented in a symptom-related framework (i.e. when participants were asked to rate perceived dyspnea, rather than perceived breathing intensity) and only in the recovery phase, when physiological input was low in detail $(10,11)$. It is thought that findings obtained using both these paradigms demonstrate an altered balance between topdown and bottom-up influences in the eventual symptom experience (i.e. "distorted symptom perception". This altered balance is thought to result from the combination of reduced detail in sensory-perceptual processing and a heightened (not necessarily conscious) expectation of symptoms in high HSR/FSS patients $(4,5,12,13)$.

Recently, we replicated both these findings in a large well-defined sample of patients with fibromyalgia and/or CFS, as part of a larger project investigating inter-individual differences in symptom perception processes in FSS. As expected, we demonstrated that FSS patients, on average, 1) had higher increases in symptom reporting after the induction of negative affective 
states than healthy controls 2) recovered more slowly from induced dyspnea than healthy controls, while there were no group-related differences in physiological measures of respiration. However, these studies also demonstrated large inter-individual variability within the patient group. Since we assume that the processes underlying these group-level results are similar for both paradigms, the primary purpose of this report is to investigate whether those patients that are sensitive to the affective modulation of symptom reporting (as assessed by the picture viewing paradigm) also have distorted perception of induced dyspnea (as assessed by the rebreathing paradigm). The second purpose of this report is to describe which patients are sensitive to this presumed altered balance between top-down and bottom-up influences, i.e. which patient characteristics are related to distorted symptom perception as assessed by both these paradigms. For example, in the picture paradigm, we observed a moderating role of difficulty identifying feelings and absorption on the affective modulation of symptom reporting (12). In the rebreathing paradigm, we found a moderating role of somatic symptom severity on the delayed recovery of symptoms despite equal recovery in physiological response (13). These individual difference variables were chosen a priori based on well-grounded hypotheses. Since both paradigms were run in the same large group of FSS patients, we want to further explore the correspondence between the findings in the picture paradigm and those in the rebreathing paradigm. In addition, we will also explore other individual difference variables that were measured in the context of the larger project (see below). The data related to this second aim are based on post-hoc unplanned analyses and should therefore be treated as exploratory findings.

\section{Methods}

\section{Patients}

Patients diagnosed with fibromyalgia and/or CFS were recruited through the psychiatry departments of the ZOL Limburg hospital (Genk, Belgium) and University Hospitals Leuven 
(Leuven, Belgium), through the Rheumatology center in Genk (Belgium) and through different patient organizations. Only patients with a clinical diagnosis were included in the study. Further, patients filled in questionnaires checking for the 2010 ACR criteria for fibromyalgia (14) and for the 1994 CDC criteria for CFS (15). Patients were excluded from participation if they had anorexia or bulimia nervosa, alcohol - or drug dependency, (history of) psychosis and any comorbid chronic cardiovascular, neurological or respiratory disorders. Other psychiatric comorbidities, such as depression and anxiety disorders, were not an exclusion criterion but were assessed by means of the MINI International Neuropsychiatric Interview (see further) to allow for a sample representative of the fibromyalgia/CFS population. The study was approved by the Medical Ethical Committees of ZOL Limburg hospital and University Hospitals Leuven.

\section{Symptom perception measures}

Affective modulation of symptom reporting was measured by means of a picture viewing paradigm. Detailed methods are published elsewhere (12). Briefly, patients filled out a symptom checklist after viewing a negative, positive, and a neutral picture series in order to investigate to what extent participants tend to report more symptoms during negative emotional states. We previously published results from this study indicating that negative emotional states enhance symptom reports in the patient group, but not in an age-and gender matched healthy control group. Further, we reported that within the patient group, this effect was moderated by "difficulty to identify feelings", as measured by the subscale of the Toronto Alexithymia Scale, and by the tendency to get absorbed in experiences (12). In order to explore the relationship between distorted symptom perception in this paradigm and in the rebreathing paradigm, a symptom difference score was calculated by subtracting the symptoms reported after watching the positive and neutral picture series (average of the two) from the symptoms reported after watching the negative picture series. The symptom difference score is considered a quantification of the extent to which symptom reporting is increased during negative emotional 
states. Thus, a more extreme positive score represents a larger influence of negative affective states on symptom perception. This score was logarithmically transformed before further analysis because of a strong positive skew.

Perception of induced dyspnea was investigated with a rebreathing paradigm. Detailed methods are described elsewhere (13). Briefly, participants went through a breathing trial, consisting of a 60-second baseline phase (room air), a 150-second rebreathing phase (rebreathing through a bag initially filled with a gas mixture of $95 \% \mathrm{O}_{2}$ and $5 \% \mathrm{CO}_{2}$ ), causing a progressive increase in respiratory flow, $\mathrm{PCO}_{2}$ and experienced dyspnea, and a 150 -second recovery phase (room air). Participants were not aware of the timing of alterations between room air and rebreathing. Participants continuously indicated perceived dyspnea, and respiratory flow and end-tidal fractional concentration of $\mathrm{CO}_{2}\left(\mathrm{FetCO}_{2}\right)$ were measured continuously. We previously published results from this study indicating that patients report more dyspnea than healthy controls but only in the recovery phase, despite absent differences between patients and healthy controls in physiological respiratory measures, and that this effect was moderated by somatic symptom severity within the patient group (13). Therefore, reported dyspnea during the recovery phase was considered the main outcome variable for the analyses reported in the current manuscript.

\section{Individual difference variables}

To explore which individual difference variables are correlated with both symptom perception measures within the patient group, patients completed a psychiatric diagnostic interview and filled out a set of questionnaires.

Psychiatric comorbidity was assessed by the experimenter with the MINI International Neuropsychiatric Interview (MINI) 5.0.0. based on the DSM-IV (16,17). Fulfillment of the criteria for the following psychiatric disorders was assessed: depressive episode, (hypo)mania, 
panic disorder, agoraphobia, social phobia, obsessive-compulsive disorder (OCD), posttraumatic stress disorder (PTSD), generalized anxiety disorder, somatization disorder, alcohol dependency, drug dependency, psychotic disorder, anorexia nervosa, and bulimia nervosa. Patients were excluded from participation if they fulfilled criteria for one of the latter five disorders.

Positive and negative affectivity were measured with the trait version of the Positive and Negative Affect Schedule (PANAS; 18). Respondents are asked to indicate on a 5-point Likert scale to what extent they experience each of 20 emotions in daily life.

Health-related quality of life was measured with the RAND-36 (19). Physical and mental component summary scores are calculated from the 36 items, with higher scores representing better functioning (20). These summary scores are weighted scores ranging from 0-100 and are based on a Dutch healthy reference population (21). A score of 50 represents the average score of the reference population.

Somatic symptom severity was measured with the somatic symptom scale of the Patient Health Questionnaire (PHQ-15; Kroenke, Spitzer, \& Williams, 2002). Respondents are asked to indicate on a 3-point scale how much they were bothered by each of 15 prevalent symptoms in the past four weeks ( 0 : not bothered at all - 2: bothered a lot). Only the somatic symptom scale of the PHQ was used in this study.

Childhood trauma was measured with the Childhood Trauma Questionnaire (23). Respondents are asked to indicate on a 5-point Likert Scale (1: never true - 5 very often true) to what extent 25 statements regarding their childhood apply to them. A trauma sum score was calculated by summing the 25 items, and logarithmically transformed before further analysis because of a strong positive skew. 
Alexithymia was measured with the Toronty Alexythmia Scale (TAS-20; 24). The TAS-20 consists of 20 items that can be answered on a 5-point Liker scale (1: completely disagree - 5: completely agree), measuring three subscales: Difficulty Identifying Feelings (DIF), Difficulty Describing Feelings (DDF) and Externally-Oriented Thinking (EOT).

\begin{abstract}
Absorption, the tendency to get absorbed in experiences, was measured with the Tellegen Absorption Scale (25). Respondents indicate for 39 statements whether it applies (1) or doesn't apply (0) to them. Scores on the 39 items are summed. Exemplary items are "I can be deeply moved by a sunset" or "Sometimes I can change noise into music by the way I listen to it".
\end{abstract}

Interoceptive awareness was measured with the Interoceptive Awareness Questionnaire (26). Respondents indicate on a 5-point Likert scale to what extent each of 19 statements applies to them (1: completely disagree -5 : completely agree). Two factors can be distinguished: awareness of bodily sensations (IAQ-F1; e.g.: during physical activity I can always tell when my heart rate accelerates) and attention to unpleasant bodily sensations (IAQ-F2; e.g.: When my chest hurts, I tend to focus my attention on this).

Health anxiety and illness behavior were measured with the Illness Attitude Scales (IAS; 27), which consist of 29 items that are answered on a 5-point Likert scale ( 0 : no - 4: most of the time).

\title{
Procedure and design
}

The results reported here are part of a larger project investigating individual differences in symptom perception in FSS. Participants completed a psychiatric diagnostic interview over the phone, filled out a set of online questionnaires at home and participated in a test session consisting of four well-validated paradigms in the University Hospital of leuven (Leuven, 
Belgium) or ZOL Limburg hospital (Lanaken, Belgium). The test session lasted for three hours and took place from 2 p.m. until 5 p.m. for all participants. Participants received 75 euros for participating in the entire project. Participants were instructed to (a) not consume alcohol and caffeine during 24 hours prior to the test session, (b) refrain from exercising and smoking (unless this caused great discomfort) from four hours before the test session, and (c) eat a light lunch at noon. The paradigms were always administered in the same order. The first paradigm was the picture paradigm, the second paradigm was the rebreathing paradigm, the third paradigm was a fear conditioning paradigm, and the last paradigm was a counter-irritation paradigm. The paradigms were separated by a 10-minute break, in which participants viewed neutral video clips, consisting of scenes from the nature documentary Winged Migration (28). Written informed consent was obtained from all patients before the start of the project. Data collection took place between October 2014 and December 2016.

\section{Statistical analysis}

The first goal of this report was to investigate whether patients who are sensitive to the affective modulation of symptom reporting (as assessed by the picture paradigm) also have distorted perception of induced dyspnea (as assessed by the rebreathing paradigm). Average dyspnea ratings and minute ventilation during the rebreathing paradigm were calculated for every 30 second time window ( 2 time windows in the baseline phase, 5 time windows in the rebreathing phase and the recovery phase). Multiple mixed model analyses were performed on the phases separately (baseline, rebreathing and recovery) with dyspnea ratings and minute ventilation as dependent variables in separate analyses. Time (within-subject factor) and the symptom difference score extracted from the picture paradigm (between-subject factor) were used as independent variables in all analyses. To control for potential baseline differences in any of the outcome variables, dyspnea rating or minute ventilation (respectively) in the last 30 seconds of the baseline phase was added as a covariate in the analyses on the rebreathing and the recovery 
phase. Based on the hypothesis that the same processes underlie group-level results on both paradigms, we expected that a higher symptom difference score would be related to slower recovery from rebreathing, as seen by a significant interaction effect between time and the symptom difference score on dyspnea ratings in the rebreathing phase.

To explore which individual difference variables, apart from the ones reported earlier (13) are associated with the perception of induced dyspnea, the mixed model analyses described above were repeated with the individual difference variables as independent variable (instead of the symptom difference score) in separate analyses. An individual difference variable was considered to be associated with the perception of induced dyspnea in the recovery phase in case of significant main effect of this variable on dyspnea ratings, or in case of a significant time*variable interaction effect in the recovery phase.

To explore which individual difference variables, apart from the ones reported earlier (12) are associated with affective modulation of symptom reporting, mixed model analyses were performed with symptom checklist scores as the dependent variable and condition (negative vs. neutral vs. positive) and the individual difference variables as independent variables in separate analyses (one per individual difference variable). An individual difference variable was considered to be associated with the affective modulation of symptom reporting in case of a significant condition*variable interaction effect on symptom checklist score. The goal of these exploratory analyses was to identify potential moderators of symptom perception, other than the ones that were proposed a priori, as reported in $(12,13)$. The findings resulting from these analyses should thus be interpreted with caution, and should serve as guides for future research rather than definite results.

\section{Results}

\section{Sample descriptives}


81 patients (71 women) participated in the study (mean age : 42.67, SD $=10.62$ ). Thirty-nine patients $(53.42 \%)$ met the criteria for both CFS and fibromyalgia as determined by a checklist based on the 1994 CDC criteria for CFS (15) and the 2010 ACR criteria for fibromyalgia (14). Thirty-four patients (46.58\%) met the criteria for fibromyalgia, but not for CFS. Two patients met the criteria for CFS alone, and four patients did not meet the criteria for CFS or fibromyalgia as assessed by our checklist. Since those four patients did have a recent doctorbased diagnosis, they were not excluded from the study. One patient did not fill out the questionnaire battery. Data for the rebreathing paradigm could not be used for eight patients, because they stopped the breathing trial before it was finished (six), misunderstood the instructions (one) or because of technical issues (one). Respiratory flow data from the rebreathing test of eight patients could not be used due to technical malfunctions. The most common psychiatric comorbidities in the sample were depression (46.8\%), generalized anxiety disorder (54.4\%) and somatization disorder (31.6\%). Mean values, standard deviations and correlations between the individual difference variables can be found in Table 1.

Results from analyses comparing affective modulation in symptom reporting in patients versus healthy controls and results from analyses comparing the perception of induced dyspnea in patient versus healthy controls, and the relationship between these paradigms and certain a priori determined individual difference variables have been published elsewhere separately $(12,13)$. Using the same data, results relating performance on these two paradigms in the patient group, and exploratory results relating performance on these two paradigms to other individual difference variables, are reported here.

\section{Relationship between the picture paradigm and rebreathing test (Figure 1)}

No significant main effect of the symptom difference score or time* symptom difference score interaction effects on dyspnea ratings were found in the baseline phase (main effect: $F_{1,71}=$ $1.95, p=0.17$; interaction effect: $F_{1,71}=0.11, p=0.74$ ), rebreathing phase (main effect: $F_{1,70}<$ 
$0.01, \mathrm{p}>0.99$; interaction effect: $\mathrm{F}_{4,70}=0.76, \mathrm{p}=0.55$ ) or recovery phase (main effect: $\mathrm{F}_{1,70}=$ $0.16, p=0.70$; interaction effect: $\left.F_{4,70}=0.14, p=0.97\right)$. No significant main effect of the symptom difference score or time*symptom difference score interaction effects on minute ventilation were found in the baseline phase (main effect: $F_{1,64}=0.03, p=0.86$; interaction effect: $F_{1,64}=0.99, p=0.32$ ), rebreathing phase (main effect: $F_{1,70}=0.16, p=0.69$; interaction effect: $F_{4,63}=0.59, p=0.67$ ) or recovery phase (main effect: $F_{1,70}=0.01, p=0.94$; interaction effect: $F_{4,763}=1.21, p=0.32$ ), see Figure 1 .

Relationship between individual difference variables and perception of induced dyspnea (rebreathing)

Main effects of individual difference variables on dyspnea ratings in the recovery phase of the rebreathing test and individual difference variable * time interaction effects can be found in Table 2. None of the individual difference variables that were measured were associated with dyspnea ratings during the recovery phase or with recovery rate, except for age (main effect of age: $F_{1,70}=3.30, p=0.074$; time*age interaction: $\left.F_{4,70}=3.69, p=0.009\right)$, with higher age being associated with a slower recovery from rebreathing.

Relationship between individual difference variables and affective modulation of symptom reporting (picture paradigm)

Main effects of individual difference variables on symptom reporting during the picture paradigm and individual difference variable*condition interaction effects can be found in Table 2. A significant main effect of health anxiety $\left(F_{1,78}=5.10, p=0.027\right)$, illness behavior $\left(F_{1,78}=\right.$ $7.47, \mathrm{p}=0.008)$ and trait somatic symptom severity as measured by the PHQ-15 $\left(\mathrm{F}_{1,78}=26.42\right.$, $\mathrm{p}<0.001)$ on the state symptom checklist was found, indicating that patients with high health anxiety, patients displaying a lot of illness behavior and patients with high somatic symptom severity reported more symptoms during the entire experiment, regardless of the condition. Of 
all measured variables, only health anxiety was significantly associated with affective modulation of symptom reporting, as demonstrated by a significant health anxiety*condition interaction effect $\left(\mathrm{F}_{2,78}=5.45, \mathrm{p}=0.006\right)$. Follow-up analyses indicated that the difference in symptoms reported after the negative versus neutral or positive picture series became larger with increasing health anxiety scores (Figure 2).

\section{Discussion}

The main goal of the current report was to describe the relationship between the responses to two paradigms measuring distortions in symptom perception in a well-described sample of patients with fibromyalgia and/or CFS. In a picture paradigm, it is typically shown that persons with HSR and patients with FSS report enhanced somatic symptoms after viewing pictures with negative valence. In the rebreathing paradigm, HSR and FSS patients show enhanced dyspnea reports that are less related to actual physiological changes. The first question that we wanted to address is whether enhanced symptom reporting in one paradigm is related to enhanced symptom reporting in another paradigm. Recent theories on symptom perception suggest that the perception of the internal state of the body is the end-result of an inferential process: afferent interoceptive input is perceived and modified in the light of expectations, or "priors", in Bayesian statistical inference, about the cause of the input, in a hierarchical prediction error minimization process, where prediction errors are the neural activity representing input not predicted by the prior $(4,29)$. Depending on the precision of prediction errors related to somatosensory input and the precision of the prior, the eventual percept might be determined relatively more by the prior or by the prediction errors. In situations with vague somatosensory input (imprecise prediction errors) and high (statistical) confidence in a prior, the somatic experience generated by the brain will resemble the prior more than the actual afferent input $(4,5)$. It is thought that the increase in symptom reporting following negative affective states and impaired subjective recovery from rebreathing, observations consistently made in high 
HSR and FSS patients (7-13), are both the result of high confidence in inaccurate symptomrelated priors and imprecise prediction errors $(4,12,13)$.

The results presented in this report suggest that there is no relationship between the symptom difference score emerging in the picture paradigm (the increase in symptoms after negative vs neutral or positive affect induction) and patients' dyspnea perception during recovery from rebreathing. Thus, biased symptom reporting in one paradigm is unrelated to biased symptom reporting in the other paradigm. As stated above, distortions in symptom perception may arise in a situation where highly confident symptom-related priors are activated while the prediction errors related to the afferent input are imprecise. This means that there may not only be large inter-individual variability in the accordance between perceived symptoms and physiological dysfunction, but also large intra-individual variability across different situations. The manipulations used in the picture viewing paradigm and rebreathing paradigm inherently create different external and internal contexts for a number of reasons.

First of all, the rebreathing paradigm draws on explicit symptom induction of respiratory symptoms by means of $\mathrm{CO}_{2}$-inhalation, while no physical manipulations except for affective states are used in the picture viewing paradigm.

Second, in the rebreathing paradigm only one specific symptom, i.e. dyspnea, is measured, while in the picture paradigm patients are prompted for the experience of a whole range of somatic symptoms, of which the sum score is then calculated. Patients with fibromyalgia and/or CFS who do not have experience with dyspnea or might not be threatened by dyspnea, might not have highly confident specific dyspnea-related priors. In the picture viewing paradigm, however, there is room for a wide variety of symptoms to be experienced and rated, so that there is a large chance that one or more of the symptoms are relevant for a patient. Unfortunately, we did not collect any information on patients' previous experiences with dyspnea or the perceived threat level of dyspnea, which would have enabled us to test this 
hypothesis. The only approximation of past dyspnea experience available to us was the "shortness of breath" item in the PHQ-15, which inquires about the patients' experience with dyspnea over the past four weeks. Running an additional exploratory analysis using this item as a predictor for self-reported recovery from dyspnea induction did not yield any significant results, suggesting that perception of induced dyspnea is not influenced by the experience of dyspnea in the past four weeks. However, scores on this item might be an inaccurate approximation of the precision of dyspnea-related priors, since four weeks is a relatively short amount of time.

Third, symptom perception also depends on the precision of prediction errors resulting from the afferent input. It is currently not well documented if distorted interoception in one domain (e.g. respiration) necessarily implies interoception in another bodily domain. Since the signalto-noise ratio of interoceptive input may arise at different levels of the body-to-brain axis, it can be assumed that there is both shared and unique variance in the precision of interoceptive input across modalities. Third, while symptom perception is always to some extent a process involving emotional-motivational components, the picture viewing paradigm specifically investigates the effect of negative emotional states on symptom perception. Consequently, especially patients who have difficulties with emotion regulation will stray further away from average responding of healthy controls in the picture paradigm, while these factors may have a smaller influence in the rebreathing paradigm. This is consistent with the finding that the personality trait "difficulty identifying feelings" is positively related to increased symptom reporting following negative affective states (12), but is unrelated to distorted perception of induced dyspnea. In sum, the results from this study indicate that both paradigms capture different aspects of symptom perception, and patients who experience a larger variety of and more severe symptoms in negative affective states do not necessarily have distorted perception of induced dyspnea. 
The second goal of this study was to describe (post-hoc) which individual difference variables are related to symptom perception as measured by the two paradigms within the patient group (apart from the ones that we had an a priori hypothesis about which were reported 12,13). The findings resulting from these analyses are thus meant to serve as guides for future research. Of all the variables measured, only "somatic symptom severity", as measured by the PHQ-15, was related to impaired recovery from induced dyspnea (previously reported in 13), as well as age, with older patients showing impaired recovery from induced dyspnea. None of the other variables that were expected to be related to symptom perception because of their potential impact on the precision of symptom-related priors (such as health anxiety and health-related quality of life) predicted subjective dyspnea experience during recovery from rebreathing. As stated above, this might be explained by the specificity of the symptom prompted for during the rebreathing paradigm (i.e. "dyspnea"). With regards to the picture paradigm, it was demonstrated that within the patient sample, higher health anxiety was positively related to larger increases in symptom reporting after the induction of negative affective states. This might be because patients with higher health anxiety have more confident symptom-related priors, reinforced by continuous body scanning and ruminating about physical symptoms. Interestingly, the descriptive analyses (Table 1) also revealed a high positive correlation between health anxiety and the DIF-scale of the Toronto Alexithymia Scale, indicating that patients with higher health anxiety also tend to have more difficulties in identifying their feelings. The results concerning the relationship between individual difference variables and the symptom perception measures are exploratory and should be interpreted with caution, though the results presented in this manuscript warrant further research on the relationship between health anxiety and the affective modulation of symptom reporting.

In conclusion, the current results suggest that biased symptom reporting in one paradigm is unrelated to biased symptom reporting in the other paradigm, indicating that distorted symptom 
perception in FSS patients is not a trait-like, cross-situationally stable condition, but a versatile dysfunction that is very context-dependent. This is consistent with the finding that different moderators could be identified depending on the paradigm. Given the hypothesis that symptom burden in FSS patients might be reduced by targeting distorted symptom perception (5), tailored paradigms might need to be developed in order to optimize treatment for patients with varying types of distortions in symptom perception.

\section{$\underline{\text { References }}$}

(1) Clauw DJ. Fibromyalgia: an overview. Am J Med 2009;122:S3-S13.

(2) Khalsa SS, Adolphs R, Cameron OG, Critchley HD, Davenport PW, Feinstein JS, Garfinkel S, Lane RD, Mehling WE, Meuret AE, Nemeroff CB, Oppenheimer S, Petschner FH, Pollatos O, Rhudy JL, Schraam LP, Simmons WK, Stein MB, Stephan KE, Van den Bergh O, Van Diest I, Von Leupoldt A, Paulus MP, and the Interoception Summit 2016 participants. Interoception and mental health: a roadmap. Biol Psychiatry Cogn Neurosci Neuroimaging 2018;3:501-13

(3) Van den Bergh O, Bogaerts K, Van Diest I. Symptom perception, awareness and interpretation. In: Wright JD, editor. International Encyclopedia of the Social \& Behavioral Sciences. Vol 23. 2nd ed. Oxford: Elsevier; 2015. p 866-72.

(4) Van den Bergh O, Witthöft M, Petersen S, Brown RJ. Symptoms and the body: Taking the inferential leap. Neurosci Biobehav Rev 2017; 74:185-203.

(5) Henningsen P, Gündel H, Kop WJ, Löwe B, Martin A, Rief W, Rosmalen J, Schröder A, van der Feltz-Cornelis C, Van den Bergh O. Persistent physical symptoms as perceptual dysregulation: a neuropsychobehavioral model and its clinical implications. Psychosom Med 2018; 80:422-31.

(6) Van den Bergh O, Walentynowicz M. Accuracy and bias in retrospective symptom reporting. Curr Opin Psychiatry 2016;29:302-8. 
(7) Bogaerts K, Janssens T, De Peuter S, Van Diest I, Van den Bergh O. Negative affective pictures can elicit physical symptoms in high habitual symptom reporters. Psychol Health $2010 ; 25: 685-98$.

(8) Bogaerts K, Rayen L, Lavrysen A, Van Diest I, Janssens T, Schruers K, Van den Bergh O. Unraveling the relationship between trait negative affectivity and habitual symptom reporting. PloS One 2015;10:e0115748.

(9) Constantinou E, Bogaerts K, Van Diest I, Van den Bergh O. Inducing symptoms in high symptom reporters via emotional pictures: the interactive effects of valence and arousal. J Psychosom Res 2013;74:191-6.

(10) Bogaerts K, Millen A, Li W, De Peuter S, Van Diest I, Vlemincx E, Fannes S, Van den Bergh O. High symptom reporters are less interoceptively accurate in a symptom-related context. J Psychosom Res 2008;65: 417-24.

(11) Bogaerts K, Van Eylen L, Li W, Bresseleers J, Van Diest I, De Peuter S, Stans L, Decramer M, Van den Bergh O. Distorted symptom perception in patients with medically unexplained symptoms. J Abnorm Psychol 2010;119:226-34.

(12) Van Den Houte M, Bogaerts K, Van Diest I, De Bie J, Persoons P, Van Oudenhove L, Van den Bergh O. Inducing somatic symptoms in functional syndrome patients: effects of manipulating state negative affect. Psychosom Med 2017;79:1000-7.

(13) Van Den Houte M, Bogaerts K, Van Diest I, De Bie J, Persoons P, Van Oudenhove L, Van den Bergh O. Perception of induced dyspnea in fibromyalgia and chronic fatigue syndrome. J Psychosom Res 2018; 106:49-55.

(14) Wolfe F, Clauw DJ, Fitzcharles M-A, Goldenberg DL, Katz RS, Mease P, Russel AS, Russel IJ, Winfield JB, Yunus MB. The American College of Rheumatology preliminary diagnostic criteria for fibromyalgia and measurement of symptom severity. Arthritis Care Res 2010;62:600-10. 
(15) Fukuda K, Straus S, Hickie I, Sharpe M, Dobbins J, Komaroff A. The chronic fatigue syndrome: a comprehensive approach to its definition and study. Ann Intern Med 1994;121:953-9.

(16) Overbeek T, Schruers K, Griez E. MINI International Neuropsychiatric Interview, Dutch version 5.0.0 (DSM-IV). Maastricht: University of Maastrich 1999.

(17) Sheehan DV, Lecrubier Y, Sheehan KH, Amorim P, Janavs J, Weiller E, Hergueta T, Baker MS, Dunbar GC. (1998). The Mini-International Neuropsychiatric Interview (M.I.N.I.): The development and validation of a structured diagnostic psychiatric interview for DSM-IV and ICD-10. J Clin Psychiatry 1998;59:22-33.

(18) Watson D, Clark LA, Tellegen A (1988). Development and validation of brief measures of positive and negative affect: the PANAS scales. J Pers Soc Psychol 1988;54:1063-70.

(19) Hays RD, Sherbourne CD, Mazel RM. The RAND 36-item health survey 1.0. Health Econ 1993;2:217-27.

(20) Ware JE, Kosinski M, Keller SD. Physical and mental health summary scales-a user's manual. New England Medical Center. The Health Institute, Boston, MA 1994.

(21) Aaronson NK, Muller M, Cohen PDA, Essink-Bot, M-L, Fekkes M, Sanderman R, Sprangers MAG, te Velde A, Verrips E. Translation, validation, and norming of the Dutch language version of the SF-36 Health Survey in community and chronic disease populations. J Clin Epidemiol 1998;51:1055-68.

(22) Kroenke K, Spitzer RL, Williams JBW. The PHQ-15: validity of a new measure for evaluating the severity of somatic symptoms. Psychosom Med 2002;64:258-66.

(23) Scher C, Stein M, Asmundson G, McCreary D, Forde D. The childhood trauma questionnaire in a community sample: psychometric properties and normative data. $\mathbf{J}$ Trauma Stress 2001;14:843-57. 
(24) Bagby M, Parker JDA, Taylor GJ. The twenty-item Toronto Alexithymia Scale - I. Item selection and cross-validation of the factor structure. J Psychosom Res 1994;38:2332.

(25) Tellegen A. Content categories: absorption items (Revised). Unpublished manuscript, University of Minnesota 1982.

(26) Bogaerts K, Walentynowizc M, Van Den Houte M, Constantinou E, Van den Bergh O. (2018). The Interoceptive Awareness Questionnaire (IAQ) differentiates between and within groups with stress-related bodily complaints versus healthy controls. Manuscript in preparation, 2018.

(27) Kellner R. Abridged manual of the illness attitude scales. University of New Mexico, Department of Psychiatry, School of Medicine 1987.

(28) Barratier C (Producer), Perrin J (Director). Winged migration [Motion Picture]. France: Sony Pictures Classics 2001. (n.d.). Elsevier Inc.

(29) Edwards MJ, Adams RA, Brown H, Paree I, Friston KJ. A Bayesian account of “hysteria". Brain 2012;11:3495-512. 

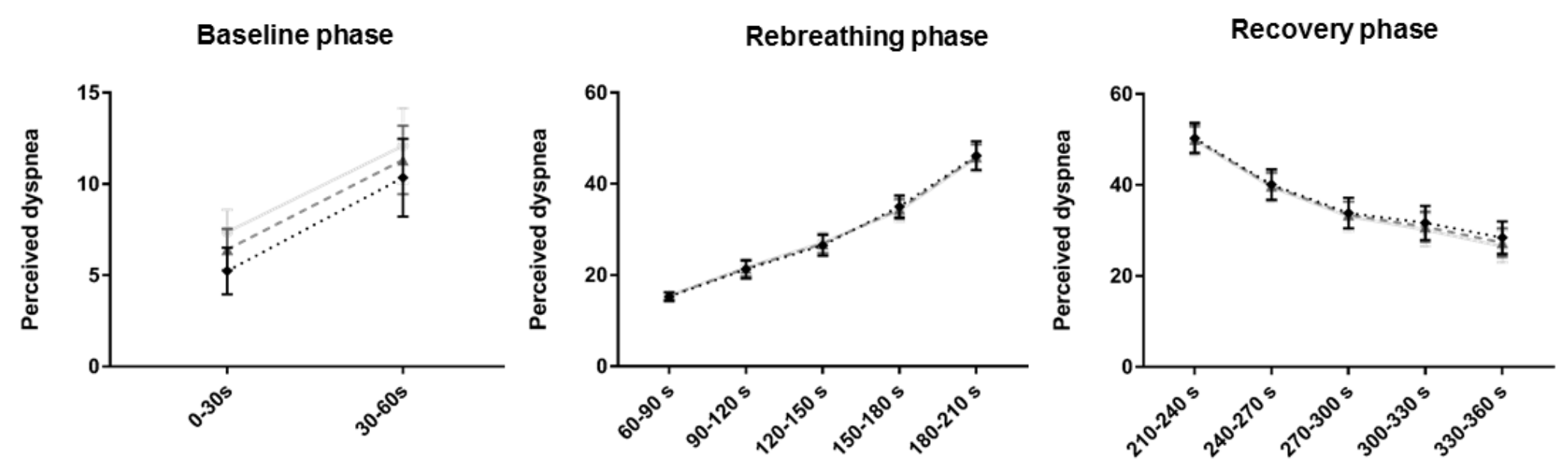

Symptom difference score

-25th percentile

-...- 50th percentile

.... 75th percentile
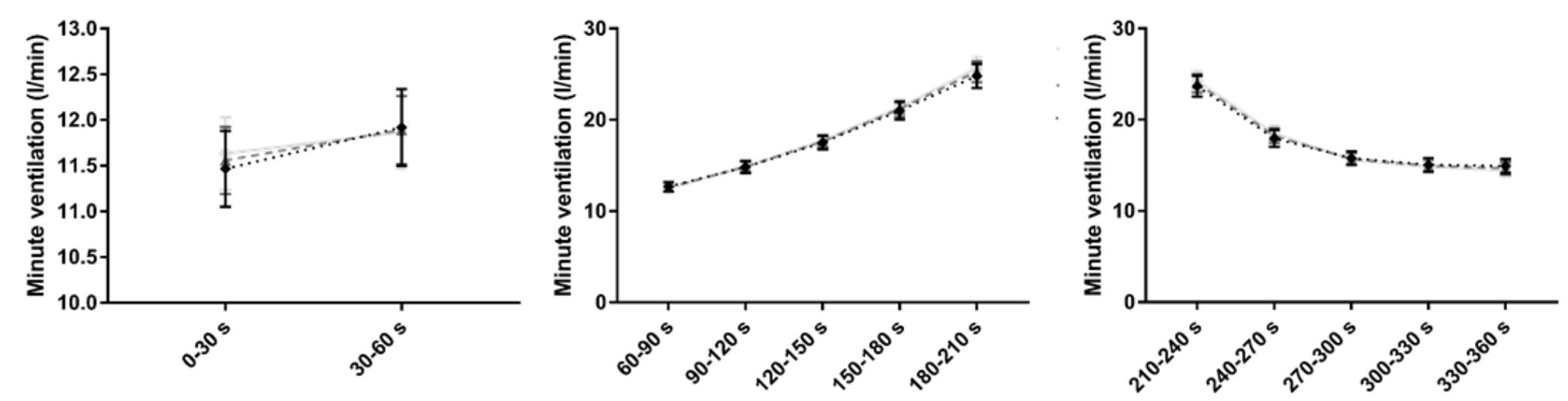

Symptom difference score

-25th percentile

- $1--50$ th percentile

$\cdots \cdots$ 75th percentile

Figure 1 


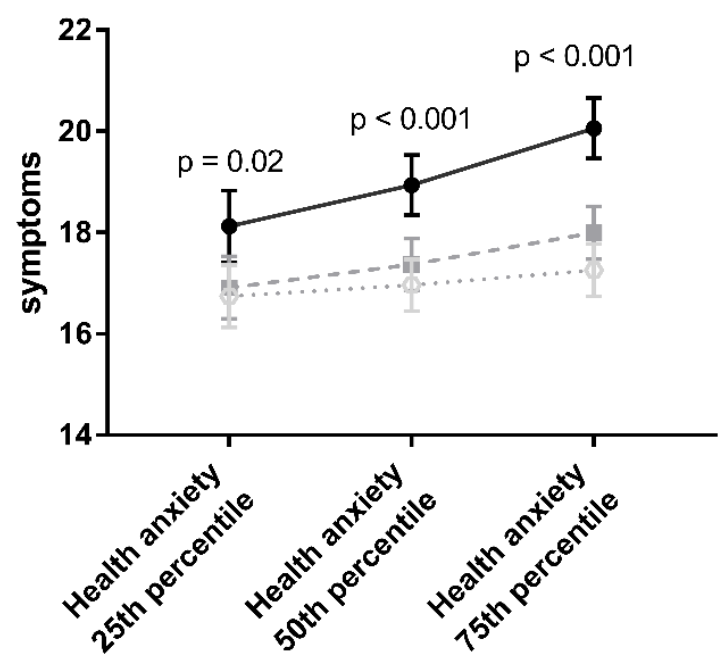

- negative affective pictures

- - neutral affective pictures

positive affective pictures

Figure 2 


\section{Figure Legends}

Figure 1. Average dyspnea ratings and minute ventilation during the baseline, rebreathing and recovery phase of the rebreathing paradigm by symptom difference score as calculated from the picture paradigm. Error bars denote standard errors.

Figure 2. Interaction effect between health anxiety (continuous variable measured by the Illness Attitude Scales) and picture category (neutral vs. positive. vs. negative) on symptom ratings after picture viewing. Error bars denote standard errors. P-values reflect main effects of the condition on symptom checklist scores at different levels of health anxiety. 